\title{
On perturbations of Hilbert spaces and probability algebras with a generic automorphism ${ }^{1}$
}

\author{
ITAÏ BEN YAACOV \\ ALEXANDER BERENSTEIN
}

\begin{abstract}
We prove that $I H S_{A}$, the theory of infinite dimensional Hilbert spaces equipped with a generic automorphism, is $\aleph_{0}$-stable up to perturbation of the automorphism, and admits prime models up to perturbation over any set. Similarly, $A P r_{A}$, the theory of atomless probability algebras equipped with a generic automorphism is $\aleph_{0}$-stable up to perturbation. However, not allowing perturbation it is not even superstable.
\end{abstract}

2000 Mathematics Subject Classification 03C45 (primary); 03C90, 03 C95 (secondary)

Keywords: Hilbert space, probability algebra, automorphism, perturbation, stability

\section{Introduction}

It was proved by Chatzidakis and Pillay [11] that if $T$ is a first order superstable theory, and the theory $T_{\tau}=T \cup\{\tau$ is an automorphism $\}$ has a model companion $T_{A}$, then $T_{A}$ is supersimple. Throughout this paper we refer to $T_{A}$ (when it exists) as the theory of models of $T$ equipped with a generic automorphism.

Continuous first order logic is an extension of first order logic, introduced in [7] as a formalism for a model theoretic treatment of metric structures (see also [6] for a general exposition of the model theory of metric structures). It is a natural question to ask whether the theorem of Chatzidakis and Pillay generalises to continuous logic and metric structures.

The proof of Chatzidakis and Pillay would hold in metric structures if we used the classical definitions of superstability and supersimplicity literally (namely, types do not fork over finite sets). These definitions, however, are known to be too strong in

\footnotetext{
${ }^{1}$ Research supported by NSF grant DMS-0500172, by ANR chaire d'excellence junior THEMODMET (ANR-06-CEXC-007) and by Marie Curie research network ModNet
} 
metric structures, and need to be weakened somewhat in order to make sense. For example, the theory of Hilbert spaces has a countable language, is totally categorical and does not satisfy the classical definition of superstability.

The standard definition for $\aleph_{0}$-stability and superstability for metric structures ([14], and later [2]) comes from measuring the size of a type space not by its cardinality but by its density character in the metric induced on it from the structures. A continuous theory is supersimple if for every $\varepsilon>0$, the $\varepsilon$-neighbourhood of a type does not fork over a finite set of parameters, or equivalently, if ordinal Lascar ranks corresponding to " $\varepsilon$-dividing" exist. A theory is superstable if and only if it is stable and supersimple. Similarly, $\aleph_{0}$-stability is equivalent to the existence of ordinal $\varepsilon$-Morley ranks, which may be defined via a metric variant of the classical Cantor-Bendixson ranks (see [4] for a general study of such ranks).

With these corrected definitions, the class of $\aleph_{0}$-stable theories is rich with examples: Hilbert spaces, probability algebras, $L^{p}$ Banach lattices and so on. Furthermore, many classical results can be generalised. For example, an $\aleph_{0}$-stable theory has prime models over every set, an uncountably categorical theory in a countable language is $\aleph_{0}$-stable, and so on (see [2]). A somewhat more involved preservation result was shown by the first author [3], namely that the theory of lovely pairs of models of a supersimple (respectively, superstable) theory is again supersimple (respectively, superstable).

With superstability and supersimplicity defined as above, the question whether a superstable theory with a generic automorphism is supersimple arises again. A specific instance of this question was asked by the second author and C. Ward Henson [10] regarding the theory of probability algebras equipped with a generic automorphism. It was answered negatively by the first author, showing that probability algebras with a generic automorphism are not superstable. The proof appears in Section 3.

However, the notions of $\aleph_{0}$-stability and/or superstability mentioned above might still be too strong: while they consider types of tuples up to arbitrarily small distance, one may further relax this and consider types also up to arbitrarily small perturbations of the entire language, or parts thereof. This idea can be formalised with the theory of perturbations as developed in [1] and somewhat restated in [4, Section 4]. We shall assume some familiarity with the second reference.

The goal of this paper is to study carefully two examples: Hilbert spaces and probability algebras, both equipped with a generic automorphism. The theory $A P r$ of atomless probability algebras and the theory IHS of infinite dimensional Hilbert spaces have some features in common. They are $\aleph_{0}$-stable, separably categorical over any finite set 
of parameters and types over sets are stationary. It follows (see [10]) that both $I H S_{\tau}$ and $A P r_{\tau}$ admit model companions $I H S_{A}$ and $A P r_{A}$.

In Section 1 we deal with the theory $I H S_{A}$ of Hilbert spaces equipped with a generic automorphism. We recall some if its properties from [8]. We use a Corollary of the Weyl-von Neumann-Berg Theorem to show that $I H S_{A}$ is $\aleph_{0}$-stable up to perturbation (of the automorphism), and admits prime models up to perturbation over any set. Unlike the arguments in [8], our arguments can be extended to a generic action of a finitely generated group of automorphisms (i.e., a generic unitary representation, see [9]) and even to Hilbert spaces equipped with a generic action of a fixed finitely generated $C^{*}$-algebra. This section also serves as a soft analogue for the main results of the other sections.

In Section 2 we deal with the theory $A \operatorname{Pr}_{A}$ of probability algebras with a generic automorphism, first studied in [10]. Specifically, we show that $\operatorname{APr}_{A}$ is $\aleph_{0}$-stable up to perturbations of the automorphism. It is an open question if $A P r_{A}$ admits prime models up to perturbations.

In Section 3 we conclude with the first author's proof that without perturbation the theory $\mathrm{APr}_{A}$ is not superstable, showing that the results of Section 2 are in some sense optimal.

\section{Hilbert spaces with an automorphism}

Let us consider a Hilbert space $\mathcal{H}$ and let $B(\mathcal{H})$ denote the space of bounded linear operators on $\mathcal{H}$. We recall that the operator norm of $T \in B(\mathcal{H})$ is $\|T\|=\sup _{\|x\|=1}\|T(x)\|$. We also recall the notions of the spectrum, punctual spectrum and essential spectrum of an operator $T \in B(\mathcal{H})$ :

$$
\begin{aligned}
\sigma(T) & =\{\lambda \in \mathbb{C}: T-\lambda I \text { is not invertible }\}, \\
\sigma_{p}(T) & =\{\lambda \in \mathbb{C}: \operatorname{ker}(T-\lambda I) \neq 0\}, \\
\sigma_{e}(T) & =\{\text { non isolated points of } \sigma(T)\} \cup\{\lambda \in \mathbb{C}: \operatorname{dim} \operatorname{ker}(T-\lambda I)=\infty\} .
\end{aligned}
$$

Definition 1.1 Let $\mathcal{H}$ be a Hilbert space, $T_{0}, T_{1} \in B(\mathcal{H})$. We say that $T_{0}$ and $T_{1}$ are approximately unitarily equivalent if there is a sequence of unitary operators $\left\{U_{n}\right\}_{n \in \mathbb{N}}$ such that $\left\|T_{0}-U_{n} T_{1} U_{n}^{*}\right\| \rightarrow 0$.

Fact 1.2 (Weyl-von Neumann-Berg Theorem [12, p. 60]) Let $\mathcal{H}$ be a Hilbert space and let $T_{0}, T_{1} \in B(\mathcal{H})$ be normal operators. Then $T_{0}$ and $T_{1}$ are approximately unitarily equivalent if and only if 
(i) $\sigma_{e}\left(T_{0}\right)=\sigma_{e}\left(T_{1}\right)$

(ii) $\operatorname{dim} \operatorname{ker}\left(T_{0}-\lambda I\right)=\operatorname{dim} \operatorname{ker}\left(T_{1}-\lambda I\right)$ for all $\lambda$ in $\mathbb{C} \backslash \sigma_{e}\left(T_{0}\right)$.

When considering a Hilbert space as a continuous structure we shall replace it with its unit ball, as described in [5]. We shall use the language $\mathcal{L}=\left\{0,-, \dot{2}, \frac{x+y}{2}\right\}$, where $\dot{2} x=\min \left(2, \frac{1}{\|x\|}\right) x$ and $d(x, y)=\left\|\frac{x-y}{2}\right\|$. Notice that we can recover the norm as $\|x\|=d(x,-x)$. An axiomatisation for the class of (unit balls of) Banach spaces in this language, excluding the symbol $\dot{2}$, appears in [5]. The symbol 2 serves as a Skolem function for the fullness axiom there, yielding a universal theory. A Banach space is a Hilbert space if and only if the parallelogram identity holds, which is a universal condition as well:

$$
\|x+y\|^{2}+\|x-y\|^{2}=2\|x\|^{2}+2\|y\|^{2}
$$

We obtain that the class of Hilbert spaces is elementary, admitting a universal theory $H S$. Its model companion is $I H S$, the theory of infinite dimensional Hilbert spaces, obtained by adding the appropriate scheme of existential conditions. It is easy to check that the theory $H S$ has the amalgamation property, so IHS eliminates quantifiers (i.e., it is the model completion of $H S$ ).

Now let $\tau$ be a new unary function symbol and let $\mathcal{L}_{\tau}$ be $\mathcal{L} \cup\{\tau\}$. Let $I H S_{\tau}$ be the theory IHS $\cup\{\tau$ is an automorphism $\}$. Since IHS is $\aleph_{0}$-stable and separably categorical even after naming finitely many constants, the theory $I H S_{\tau}$ admits a model companion $I H S_{A}$ (see [10]). The universal part of $I H S_{\tau}$ is $\left(I H S_{\tau}\right)^{\forall}=\left(H S_{\tau}\right)^{\forall}=$ $H S \cup\{\tau$ is a linear and isometric $\}$. It is again relatively easy to check that $\left(H S_{\tau}\right)^{\forall}$ has the amalgamation property. Indeed, if $\left(\mathcal{H}_{0}, \tau_{0}\right) \subseteq\left(\mathcal{H}_{i}, \tau_{i}\right)$ for $i=1,2$ then we may write $\left(\mathcal{H}_{i}, \tau_{i}\right)=\left(\mathcal{H}_{0}, \tau_{0}\right) \oplus\left(\mathcal{H}_{i}^{\prime}, \tau_{i}^{\prime}\right)$, where $\oplus$ is the orthogonal direct sum, and then $\left(\mathcal{H}_{0}, \tau_{0}\right) \oplus\left(\mathcal{H}_{1}^{\prime}, \tau_{1}^{\prime}\right) \oplus\left(\mathcal{H}_{2}^{\prime}, \tau_{2}^{\prime}\right)$ will do. It thus follows that $I H S_{A}$ eliminates quantifiers as well.

Proposition 1.3 (Ben Yaacov, Usvyatsov, Zadka [8]) Let $\mathcal{H}$ be a separable Hilbert space and let $\tau$ be a unitary operator on $\mathcal{H}$. Then $(\mathcal{H}, \tau) \vDash I H S_{A}$ (i.e., $(\mathcal{H}, \tau)$ is existentially closed as a model of $I H S_{\tau}$ ) if and only if $\sigma(\tau)=S^{1}$.

Proof Clearly, if $(\mathcal{H}, \tau)$ is existentially closed, then $\sigma(\tau)=S^{1}$. On the other hand, assume that $(\mathcal{H}, \tau) \vDash I H S_{\tau}$ and that $\sigma(\tau)=S^{1}$. Passing to an elementary substructure, we may assume that $\mathcal{H}$ is separable. Now let $\left(\mathcal{H}_{0}, \tau_{0}\right)$ be separable and existentially closed. Since $I H S$ is separably categorical, we may assume that $\mathcal{H}_{0}=\mathcal{H}$. Since $\sigma\left(\tau_{0}\right)=\sigma(\tau)=S^{1}$, by Fact 1.2 there is a sequence $\left\{U_{n}\right\}_{n \in \omega}$ of unitary operators on 
$\mathcal{H}$ such that $U_{n} \tau_{1} U_{n}^{*} \rightarrow \tau_{0}$ in norm. It follows that if $\mathcal{U}$ is a non-principal ultra-filter on $\mathbb{N}$ then $\Pi_{\mathcal{U}}\left(\mathcal{H}, U_{n} \tau U_{n}^{*}\right)=\Pi_{\mathcal{U}}\left(\mathcal{H}, \tau_{0}\right)$.

On the other hand, $\left(\mathcal{H}, U_{n} \tau U_{n}^{*}\right) \cong(\mathcal{H}, \tau)$ for all $n \in \mathbb{N}$. Thus $\Pi_{\mathcal{U}}(\mathcal{H}, \tau) \cong \Pi_{\mathcal{U}}\left(\mathcal{H}, \tau_{0}\right)$, whereby $(\mathcal{H}, \tau) \equiv\left(\mathcal{H}, \tau_{0}\right) \vDash I H S_{A}$.

Remark 1.4 Henson and Iovino observed that the theory $I H S_{A}$ is not $\aleph_{0}$-stable (or even small) in the sense defined in the introduction. Indeed, let $(\mathcal{H}, \tau) \vDash I H S_{A}$ be $\aleph_{1}$-saturated and for each $\lambda \in S^{1}$ let $v_{\lambda} \in H$ be a normal vector such that $\tau v_{\lambda}=\lambda v_{\lambda}$. Then $d\left(\operatorname{tp}\left(v_{\lambda}\right), \operatorname{tp}\left(v_{\rho}\right)\right)=\sqrt{2}$ for $\lambda \neq \rho$. Thus the metric density character of $\mathrm{S}_{1}(\varnothing)$ is the continuum.

On the other hand, it is shown in [8] that $I H S_{A}$ is superstable.

Let $\operatorname{dcl}_{\tau}$ and $\operatorname{acl}_{\tau}$ denote the definable and algebraic closure (in the real sort) in models of $I H S_{A}$. We claim that if $(\mathcal{H}, \tau) \vDash I H S_{A}$ and $A \subseteq H$, then $\operatorname{dcl}_{\tau}(A)=$ $\operatorname{acl}_{\tau}(A)=\operatorname{dcl}\left(\bigcup_{n \in \mathbb{Z}} \tau^{n}(A)\right)$, where $\operatorname{dcl}(A)$ is the definable closure of $A$ in the language $\mathcal{L}$. Indeed, let $B=\operatorname{dcl}\left(\bigcup_{n \in \mathbb{Z}} \tau^{n}(A)\right)$. Then clearly $B \subseteq \operatorname{dcl}_{\tau}(A)$. On the other hand, we may decompose $(\mathcal{H}, \tau)=\left(B, \tau \uparrow_{B}\right) \oplus\left(B^{\prime}, \tau \uparrow_{B^{\prime}}\right)$, in which case $(\mathcal{H}, \tau) \preceq$ $\left(B, \tau \uparrow_{B}\right) \oplus \bigoplus_{n \in \mathbb{N}}\left(B^{\prime}, \tau \uparrow_{B^{\prime}}\right)$, showing that $\operatorname{acl}_{\tau}(A) \subseteq B$.

We may similarly characterise non forking in models of $I H S_{A}$. For $(\mathcal{H}, \tau) \vDash I H S_{A}$ and subsets $A, B, C \subseteq \mathcal{H}$, say that $A \downarrow_{B} C$ if $P_{\mathrm{dcl}_{\tau}(B)}(a)=P_{\mathrm{dcl}_{\tau}(B C)}(a)$ for every $a \in A$. We leave it to the reader to check that $\downarrow$ satisfies the usual axioms of a stable notion of independence (invariance, symmetry, transitivity, and so on), and therefore coincides with non forking.

Proposition 1.5 Let $(\mathcal{H}, \tau) \vDash I H S_{A}, A, B \subseteq \mathcal{H}$. Then $\operatorname{tp}(A / B)$ is stationary and $\mathrm{Cb}(A / B)$ is inter-definable with the set $\left.C=\left\{P_{\operatorname{dcl}_{\tau}(B)}(a)\right\}\right)_{a \in A}$.

Proof Stationarity follows from the characterisation of independence (and from quantifier elimination). It is also clear that $C \subseteq \mathrm{dcl}_{\tau}(B)$ and $A \downarrow_{C} B$, and since we already know that $\operatorname{tp}(A / C)$ is stationary as well, we obtain $\mathrm{Cb}(A / B) \subseteq C$.

For the converse it suffices to show that for every $a \in A$, the projection $P_{\mathrm{dcl}_{\tau}(B)}(a)$ belongs to the definable closure of any Morley sequence in $\operatorname{tp}(A / B)$. So let $\left(A_{n}\right)_{n \in \mathbb{N}}$ be such a Morley sequence. Then $P_{\mathrm{dcl}_{\tau}(B)}\left(a_{n}\right)=P_{\mathrm{dcl}_{\tau}(B)}(a)$ for all $a \in A$ and all $n$, so $\left\{a_{n}-P_{\mathrm{dcl}_{\tau}(B)}(a)\right\}_{n \in \mathbb{N}}$ forms an orthogonal sequence of bounded norm. Thus

$$
\sum_{n=0}^{m-1} \frac{a_{n}}{m}=P_{\operatorname{dcl}_{\tau}(B)}(a)+\sum_{n=0}^{m-1} \frac{a_{n}-P_{\mathrm{dcl}_{\tau}(B)}(a)}{m} \rightarrow P_{\operatorname{dcl}_{\tau}(B)}(a) . \boldsymbol{\square}_{1.5}
$$


It follows that $I H S_{A}$ has weak elimination of imaginaries, namely that for every imaginary element $e$ there exists a real tuple (possibly infinite) $A$ such that $A \subseteq \operatorname{acl}_{\tau}(e)$, $e \in \operatorname{dcl}^{e q}(A)$.

We now turn to perturbations of the automorphism in models of $I H S_{A}$. Let $\left(\mathcal{H}_{i}, \tau_{i}\right) \vDash$ $I H S_{A}$ for $i=0,1$, and let $r \geq 0$. We define an $r$-perturbation of $\left(\mathcal{H}_{0}, \tau_{0}\right)$ to $\left(\mathcal{H}_{1}, \tau_{1}\right)$ to be an isometric isomorphism of Hilbert spaces $U: \mathcal{H}_{0} \cong \mathcal{H}_{1}$ which satisfies in addition

$$
\left\|U \tau_{0} U^{-1}-\tau_{1}\right\| \leq r .
$$

The set of all $r$-perturbations will be denoted $\operatorname{Pert}_{r}\left(\left(\mathcal{H}_{0}, \tau_{0}\right),\left(\mathcal{H}_{1}, \tau_{1}\right)\right)$. It is fairly immediate to verify that this indeed satisfies all the conditions stated in [4, Theorem 4.4], and therefore does indeed correspond to a perturbation system as defined there.

Lemma 1.6 Let $\left(\mathcal{H}_{0}, \tau_{0}\right) \subseteq\left(\mathcal{H}_{i}, \tau_{i}\right)$ be separable models of $I H S_{\tau}$ for $i=1,2$. Then we may write $\left(\mathcal{H}_{i}, \tau_{i}\right)=\left(\mathcal{H}_{0}, \tau_{0}\right) \oplus\left(\mathcal{H}_{i}^{\prime}, \tau_{i}^{\prime}\right)$, and let us assume that $\sigma\left(\tau_{1}^{\prime}\right) \subseteq \sigma\left(\tau_{2}^{\prime}\right)$ and that $\sigma\left(\tau_{1}^{\prime}\right)$ has no isolated points.

Then for every $\varepsilon>0$ there is an isometric isomorphism $U: \mathcal{H}_{1} \oplus \mathcal{H}_{2}^{\prime} \cong \mathcal{H}_{2}$, which fixes $\mathcal{H}_{0}$ such that $\left\|U\left(\tau_{1} \oplus \tau_{2}^{\prime}\right) U^{-1}-\tau_{2}\right\| \leq \varepsilon$.

Proof Under the assumptions we have $\sigma\left(\tau_{1}^{\prime} \oplus \tau_{2}^{\prime}\right)=\sigma\left(\tau_{2}^{\prime}\right)$. We also assume that $\sigma\left(\tau_{1}^{\prime}\right)$ has no isolated points. Therefore, if $\lambda \in \sigma\left(\tau_{1}^{\prime} \oplus \tau_{2}^{\prime}\right)$ is isolated then its eigenspace in $\mathcal{H}_{1}^{\prime} \oplus \mathcal{H}_{2}^{\prime}$ is entirely contained in $\mathcal{H}_{2}^{\prime}$, so the multiplicity (possibly infinite) of $\lambda$ is the same for $\tau_{1}^{\prime} \oplus \tau_{2}^{\prime}$ and for $\tau_{2}^{\prime}$. It follows that the hypotheses of Fact 1.2 hold, and we obtain $V: \mathcal{H}_{1}^{\prime} \oplus \mathcal{H}_{2}^{\prime} \cong \mathcal{H}_{2}^{\prime}$ such that $\left\|V\left(\tau_{1}^{\prime} \oplus \tau_{2}^{\prime}\right) V^{-1}-\tau_{2}^{\prime}\right\| \leq \varepsilon$. Then $U=\mathrm{id}_{\mathcal{H}_{0}} \oplus V$ will do.

Theorem 1.7 The theory $I H S_{A}$ is $\aleph_{0}$-stable up to perturbation of the automorphism.

Proof Let $\left(\mathcal{H}_{0}, \tau_{0}\right),\left(\mathcal{H}_{1}^{\prime}, \tau_{1}^{\prime}\right) \vDash I H S_{A}$ be separable, and let $\left(\mathcal{H}_{1}, \tau_{1}\right)=\left(\mathcal{H}_{0}, \tau_{0}\right) \oplus$ $\left(\mathcal{H}_{1}^{\prime}, \tau_{1}^{\prime}\right)$. By Proposition 1.3 we have $\left(\mathcal{H}_{1}, \tau_{1}\right) \vDash I H S_{A}$, so $\left(\mathcal{H}_{0}, \tau_{0}\right) \preceq\left(\mathcal{H}_{1}, \tau_{1}\right)$ by model completeness. It will therefore be enough to show that every type over $\mathcal{H}_{0}$ is realised, up to perturbation, in $\left(\mathcal{H}_{1}, \tau_{1}\right)$. Such a type can always be realised in a separable elementary extension $\left(\mathcal{H}_{2}, \tau_{2}\right) \succeq\left(\mathcal{H}_{1}, \tau_{1}\right)$. Then $\left(\mathcal{H}_{0}, \tau_{0}\right) \subseteq\left(\mathcal{H}_{2}, \tau_{2}\right)$ and we may decompose the latter as $\left(\mathcal{H}_{2}, \tau_{2}\right)=\left(\mathcal{H}_{0}, \tau_{0}\right) \oplus\left(\mathcal{H}_{2}^{\prime}, \tau_{2}^{\prime}\right)$.

Notice that $\left(\mathcal{H}_{1}^{\prime}, \tau_{1}^{\prime}\right) \subseteq\left(\mathcal{H}_{2}^{\prime}, \tau_{2}^{\prime}\right)$, so $\sigma\left(\tau_{1}^{\prime}\right)=\sigma\left(\tau_{2}^{\prime}\right)=S^{1}$. We may therefore apply Lemma 1.6, obtaining for every $\varepsilon>0$ there an isometric isomorphism $U_{\varepsilon}: \mathcal{H}_{1} \rightarrow \mathcal{H}_{2}$ fixing $\mathcal{H}_{0}$ such that $\left\|\tau_{1}-U_{\varepsilon}^{-1} \tau_{2} U_{\varepsilon}\right\|<\varepsilon$. 
We have thus shown that every type over $\mathcal{H}_{0}$ is realised, up to arbitrarily small perturbation of the automorphism, in a fixed separable extension $\left(\mathcal{H}_{1}, \tau_{1}\right) \succeq\left(\mathcal{H}_{0}, \tau_{0}\right)$, as desired.

Remark 1.8 Let $G$ be a finitely generated discrete group and let $I H S_{g G}$ be the theory of Hilbert spaces with a generic action of $G$ by automorphism (see [9]). Using Voiculescu's Theorem [12] in place of Fact 1.2, the same argument shows that the theory $I H S_{g G}$ is $\aleph_{0}$-stable up to perturbations of the automorphisms. This can even be further extended to the theory $I H S_{g \mathcal{A}}$ of a generic presentation of a finitely generated $C^{*}$-algebra $\mathcal{A}$.

Proposition 1.9 The theory $I H S_{A}$ has prime models up to perturbation over sets (of real or imaginary elements).

By this we mean that for every set $A$ in a model of $I H S_{A}$ there exists a model $\left(\mathcal{H}_{1}, \tau_{1}\right)$, containing $A$, such that if $\left(\mathcal{H}_{2}, \tau_{2}\right)$ is any other model which contains $A$ then, up to arbitrarily small perturbation of $\tau_{2}$ to $\rho_{2}$, we can embed $\left(\mathcal{H}_{1}, \tau_{1}\right)$ elementarily in $\left(\mathcal{H}_{2}, \rho_{2}\right)$ over $A$.

Proof We may assume that the set $A$ over which we seek a prime model is algebraically closed. By weak elimination of imaginaries we may assume that $A$ is a real set, and we may further assume that $A=\operatorname{dcl}_{\tau}(A)$. It is therefore a Hilbert subspace $\mathcal{H}_{0}$ (possibly finite dimensional) on which $\tau_{0}=\tau \uparrow_{\mathcal{H}_{0}}$ is an automorphism. Moreover, since $I H S_{A}$ eliminates quantifiers, the type of $\mathcal{H}_{0}$ is determined by the pair $\left(\mathcal{H}_{0}, \tau_{0}\right)$, and there is no need to consider the ambient structure.

If $\left(H_{0}, \tau_{0}\right) \vDash I H S_{A}$ there is nothing to prove. Otherwise $\sigma\left(\tau_{0}\right) \subsetneq S^{1}$. Let $\left(H_{1}^{\prime}, \tau_{1}^{\prime}\right) \vDash$ $I H S_{\tau}$ be separable such that $\sigma\left(\tau_{1}^{\prime}\right)=\overline{S^{1} \backslash \sigma\left(\tau_{0}\right)}$ (for example we may take $H_{1}^{\prime}=$ $L_{2}\left(S^{1} \backslash \sigma\left(\tau_{0}\right)\right)$ in the Lebesgue measure, with $\left.\left(\tau_{1}^{\prime} f\right)(x)=x f(x)\right)$. Let $\left(\mathcal{H}_{1}, \tau_{1}\right)=$ $\left(\mathcal{H}_{0}, \tau_{0}\right) \oplus\left(\mathcal{H}_{1}^{\prime}, \tau_{1}^{\prime}\right)$. Clearly $\sigma\left(\tau_{1}\right)=S^{1}$, so $\left(\mathcal{H}_{1}, \tau_{1}\right) \vDash I H S_{A}$, and we shall prove that it is prime, up to perturbation of the automorphism, over $\mathcal{H}_{0}$.

So let $\left(\mathcal{H}_{0}, \tau_{0}\right) \subseteq\left(\mathcal{H}_{2}, \tau_{2}\right) \vDash I H S_{A}$ and we may assume that $\mathcal{H}_{2}$ is separable. As usual, we may decompose it as $\left(\mathcal{H}_{2}, \tau_{2}\right)=\left(\mathcal{H}_{0}, \tau_{0}\right) \oplus\left(\mathcal{H}_{2}^{\prime}, \tau_{2}^{\prime}\right)$. Since $\sigma\left(\tau_{2}\right)=S^{1}$, we necessarily have $\sigma\left(\tau_{2}^{\prime}\right) \supseteq S^{1} \backslash \sigma\left(\tau_{0}\right)$, and since $\sigma\left(\tau_{2}^{\prime}\right)$ is moreover closed, it contains $\sigma\left(\tau_{1}\right)$. By Lemma 1.6, for every $\varepsilon>0$ there exists an isometric isomorphism $U: \mathcal{H}_{1} \oplus \mathcal{H}_{2}^{\prime} \cong \mathcal{H}_{2}$ fixing $\mathcal{H}_{0}$ such that $\left\|U\left(\tau_{1} \oplus \tau_{2}^{\prime}\right) U^{-1}-\tau_{2}\right\| \leq \varepsilon$. By Proposition 1.3 we also have $\left(\mathcal{H}_{1}, \tau_{1}\right) \preceq\left(\mathcal{H}_{1}, \tau_{1}\right) \oplus\left(\mathcal{H}_{2}^{\prime}, \tau_{2}^{\prime}\right)$

Thus $\rho_{2}=U\left(\tau_{1} \oplus \tau_{2}^{\prime}\right) U^{-1}$ is as desired. 


\section{Probability algebras with an automorphism}

By a probability space we mean a triplet $(X, \mathcal{B}, \mu)$, where $X$ is a set, $\mathcal{B}$ a $\sigma$-algebra of subsets of $X, \mu$ a $\sigma$-additive positive measure on $\mathcal{B}$ such that $\mu(X)=1$. A probability space $(X, \mathcal{B}, \mu)$ is called atomless if for every $A \in \mathcal{B}$ there is $C \in \mathcal{B}$ such that $\mu(A \cap C)=\frac{1}{2} \mu(A)$. We say that two elements $A, B \in \mathcal{B}$ determine the same event, and write $A \sim_{\mu} B$ if $\mu(A \triangle B)=0$. The relation $\sim_{\mu}$ is an equivalence relation and the collection of classes is denoted by $\bar{B}$ and is called the measure algebra associated to $(X, \mathcal{B}, \mu)$. Operations such as unions, interesections and complements are well defined for events, as well as the measure. The distance between two events $a, b \in \bar{B}$ is given by the measure of their symmetric difference. This renders $\overline{\mathcal{B}}$ a complete metric space.

Conversely, let $\left(\mathscr{B}, 0,1,{ }^{c}, \cup, \cap\right)$ be a Boolean algebra and assume that $d$ is a complete metric on $\mathscr{B}$. Let $\mu(x)$ be an abbreviation for $d(0, x)$ and assume furthermore that $d(x, y)=\mu(x \triangle y), \mu(x)+\mu(y)=\mu(x \cap y)+\mu(x \cup y)$ and $\mu(1)=1$. Then $\mathscr{B}$ is the probability algebra associated to some probability space (and we may moreover take that space to be the Stone space of $\mathscr{B}$, equipped with the Borel $\sigma$-algebra).

We may view probability algebras as continuous structures in the language $\mathcal{L}_{P r}=$ $\left\{0,1,{ }^{c}, \cup, \cap\right\}$ (the distance symbol is always implicit, and the measure can be recovered from it as above). The class of probability algebras is elementary and admits a universal theory denoted $\mathrm{Pr}$. Its model completion is $A P r$, the theory of atomless probability algebras. It admits quantifier elimination, is $\aleph_{0}$-categorical (even over finitely many parameters) and $\aleph_{0}$-stable (see $[7,10]$ ).

Definition 2.1 Let $\mathscr{B}$ be a probability algebra. An automorphism $\tau \in \operatorname{Aut}(\mathscr{B})$ is said to be aperiodic if for every non-zero event $a \in \mathscr{B}$ and every $n>0$ there is a sub-event $b \subseteq a$ such that $\tau^{n}(b) \neq b$. (In other words, the support of $\tau^{n}$ is 1 for all $n \geq 1$.)

Fact 2.2 (Halmos-Rokhlin-Kakutani Lemma, $[13,386 \mathrm{C}]$ ) Let $\mathscr{B}$ be a probability algebra, $\tau \in \operatorname{Aut}(\mathscr{B})$. Then $\tau$ is aperiodic if and only if, for every $n \geq 1$ and every $\varepsilon>0$ there is $a \in \mathscr{B}$ such that $a, \tau a, \ldots, \tau^{n-1}(a)$ are disjoint and $n \mu(a)>1-\varepsilon$.

Now let $\mathcal{L}_{\tau}=\mathcal{L}_{\operatorname{Pr}} \cup\{\tau\}$ where $\tau$ is a new unary function symbol. Let $A \operatorname{Pr} r_{\tau}$ be the theory $A \operatorname{Pr} \cup\{\tau$ is an automorphism $\}$. It was shown in [10] that $A P r_{\tau}$ admits a model companion $A P r_{A}$, consisting of $A P r_{\tau}$ together with axioms saying that $\tau$ is aperiodic.

Definition 2.3 By the Lebesgue space we mean the probability space $([0,1], \lambda)$, where $\lambda$ is the standard Lebesgue measure. The associated probability algebra $\mathscr{L}=$ $\mathfrak{B}([0,1], \lambda)$ is the unique separable atomless probability algebra. 
An automorphism $\tau$ of the Lebesgue space is a measurable, measure-preserving bijection between measure one subsets of $[0,1]$.

Remark 2.4 (i) Every automorphism of the probability algebra $\mathscr{L}$ comes from an automorphism of the Lebesgue space.

(ii) An automorphism $\tau$ of the Lebesgue space induces an aperiodic automorphism on $\mathscr{L}$ if and only if $\tau$ itself is aperiodic, namely if $\lambda\left\{x \in[0,1]: \tau^{n}(x)=x\right\}=0$.

Definition 2.5 Let $\mathscr{A}$ be a probability algebra. We equip $\operatorname{Aut}(\mathscr{A})$ with the uniform convergence metric

$$
d\left(\tau_{0}, \tau_{1}\right)=\sup _{x \in \mathscr{A}} d\left(\tau_{0}(x), \tau_{1}(x)\right) .
$$

Let $\left(\mathscr{A}_{i}, \tau_{i}\right) \vDash A P r_{A}$ for $i=0,1$ and let $r \geq 0$. Then an $r$-perturbation of $\left(\mathscr{A}_{0}, \tau_{0}\right)$ to $\left(\mathscr{A}_{1}, \tau_{1}\right)$ is an (isometric) isomorphism $f: \mathscr{A}_{0} \cong \mathscr{A}_{1}$ such that $d\left(f \tau_{0} f^{-1}, \tau_{1}\right) \leq r$.

Notice that this is essentially the same definition as for (unit balls of) Hilbert space. In particular, as in the Hilbert space case, this definition satisfies the conditions of [4, Theorem 4.4] and thereby comes from a perturbation system.

Definition 2.6 Let $\mathscr{A}$ be a probability algebra, $\tau \in \operatorname{Aut}(\mathscr{A}), a \in \mathscr{A}$. We say that $(a, \tau)$ generate an $(n, \varepsilon)$-partition (of $\mathscr{A})$ if $a, \tau(a), \ldots, \tau^{n-1}(a)$ are disjoint and $\mu\left(\bigvee_{i<n} \tau^{i}(a)\right) \geq 1-\varepsilon$. An $(n, 0)$-partition will simply be called an $n$-partition.

If $\mathscr{A}_{i}, i=0,1$ are probability algebras and $\mathscr{B}=\mathscr{A}_{0} \otimes \mathscr{A}_{1}$ is their free amalgam, we may identify $\mathscr{A}_{0}$ with its image $\mathscr{A}_{0} \otimes 1=\left\{a \otimes 1: a \in \mathscr{A}_{0}\right\} \subseteq \mathscr{B}$, and similarly $\mathscr{A}_{1} \cong$ $1 \otimes \mathscr{A}_{1} \subseteq \mathscr{B}$. In particular, if $\left(\mathscr{A}_{i}, \tau_{i}\right) \vDash A P r_{\tau}, i=0,1$ then $\left(\mathscr{A}_{0} \otimes \mathscr{A}_{1}, \tau_{0} \otimes \tau_{1}\right) \vDash A P r_{\tau}$ as well. If in addition $\left(\mathscr{A}_{0}, \tau_{0}\right) \vDash A P r_{A}$ then $\tau_{0}$ is aperiodic, whereby so is $\tau_{0} \otimes \tau_{1}$, i.e., $\left(\mathscr{A}_{0} \otimes \mathscr{A}_{1}, \tau_{0} \otimes \tau_{1}\right) \vDash A P r_{A}$. Since $A P r_{A}$ is model complete we conclude that

$$
\left(\mathscr{A}_{0}, \tau_{0}\right) \vDash A \operatorname{Pr}_{A},\left(\mathscr{A}_{1}, \tau_{1}\right) \vDash A P r_{\tau} \quad \Longrightarrow \quad\left(\mathscr{A}_{0}, \tau_{0}\right) \preceq\left(\mathscr{A}_{0} \otimes \mathscr{A}_{1}, \tau_{0} \otimes \tau_{1}\right) .
$$

Definition 2.7 Let $\left(\mathscr{A}, \tau_{\mathscr{A}}\right) \preceq\left(\mathscr{B}, \tau_{\mathscr{B}}\right) \vDash A P r_{A}$ be separable. We say that $\left(\mathscr{B}, \tau_{\mathscr{B}}\right)$ is partitioned over $\mathscr{A}$ if:

(i) The probability algebra $\mathscr{B}$ is isomorphic to $\mathscr{A} \otimes \mathscr{L}$ over $\mathscr{A}$ (meaning that $a \in \mathscr{A}$ gets mapped to $a \otimes 1$ ).

(ii) Under this isomorphism, for each $0<n \in \mathbb{N}$ there exists $c_{n} \in \mathscr{L}$ such that $\left(1 \otimes c_{n}, \tau_{\mathscr{B}}\right)$ generate an $n$-partition, all of whose members belong to $1 \otimes \mathscr{L}$. 
Lemma 2.8 Let $\left(\mathscr{A}, \tau_{\mathscr{A}}\right) \preceq\left(\mathscr{B}, \tau_{\mathscr{B}}\right) \vDash A P r_{A}$ be separable. Then there exists a further elementary extension $\left(\mathscr{B}, \tau_{\mathscr{B}}\right) \preceq\left(\mathscr{C}, \tau_{\mathscr{C}}\right)$ which is partitioned over $\mathscr{A}$.

Proof First of all, we may assume that $\mathscr{B}$ is atomless over $\mathscr{A}$. Indeed, $\left(\mathscr{B}^{\prime}, \tau_{\mathscr{B}}^{\prime}\right)=$ $\left(\mathscr{B}, \tau_{\mathscr{B}}\right) \otimes(\mathscr{L}$, id $)$ is a separable elementary extension of $\left(\mathscr{B}, \tau_{\mathscr{B}}\right)$ and we may replace the latter with the former. Therefore we may assume that $\mathscr{B}=\mathscr{A} \otimes \mathcal{L}$.

It is not difficult to construct an automorphism $\rho \in \operatorname{Aut}(\mathscr{L})$ such that for each $n$ there is $c_{n} \in \mathscr{L}$ such that $\left(c_{n}, \rho\right)$ generate an $n$-partition. Let $\left(\mathscr{C}, \tau_{\mathscr{C}}\right)=\left(\mathscr{B} \otimes \mathscr{L}, \tau_{\mathscr{B}} \otimes \rho\right)$, so $\left(\mathscr{B}, \tau_{\mathscr{B}}\right) \preceq\left(\mathscr{C}, \tau_{\mathscr{C}}\right)$.

On the other hand we have $\mathscr{C}=\mathscr{B} \otimes \mathscr{L}=\mathscr{A} \otimes[\mathscr{L} \otimes \mathscr{L}]$, and for every $k<n$

$$
\tau_{\mathscr{C}}^{k}\left(1 \otimes\left[1 \otimes c_{n}\right]\right)=1 \otimes\left[1 \otimes \rho^{k}\left(c_{n}\right)\right] .
$$

Now use the fact that $\mathscr{L} \otimes \mathscr{L} \cong \mathscr{L}$ to conclude.

Notation 2.9 For a probability algebra $\mathscr{C}$ and $c \in \mathscr{C}$, let $\mathscr{C}_{\leq c}$ denote the ideal $\left\{c^{\prime} \in \mathscr{C}: c^{\prime} \leq c\right\}$.

Notation 2.10 For $0<n \in \mathbb{N}$ fix $\left(\ell_{n}, \rho_{n}\right) \in \mathscr{L} \times \operatorname{Aut}(\mathscr{L})$ generating an $n$-partition such that in addition $\left(\rho_{n}\right)^{n}=\mathrm{id}$. Note that this determines $\left(\mathscr{L}, \rho_{n}\right)$ up to isomorphism, and that it is not a model of $A P r_{A}$.

Lemma 2.11 Let $\left(\mathscr{A}, \tau_{\mathscr{A}}\right) \preceq\left(\mathscr{B}, \tau_{\mathscr{B}}\right) \vDash A P r_{A}$, and assume that $\left(\mathscr{B}, \tau_{\mathscr{B}}\right)$ is partitioned over $\mathscr{A}$. Then for every $0<n \in \mathbb{N}$ there exists $\tau_{\mathscr{B}}^{\prime} \in \operatorname{Aut}(\mathscr{B})$ such that:

(i) $d\left(\tau_{\mathscr{B}}, \tau_{\mathscr{B}}^{\prime}\right) \leq \frac{1}{2 n}$.

(ii) $\left(\mathscr{B}, \tau_{\mathscr{B}}^{\prime}\right) \cong\left(\mathscr{A}, \tau_{\mathscr{A}}\right) \otimes\left(\mathscr{L}, \rho_{n}\right)$ over $\mathscr{A}$, where $\left(\mathcal{L}, \rho_{n}\right)$ are as in Notation 2.10.

Proof We may assume that $\left(\mathscr{B}, \tau_{\mathscr{B}}\right)=\left(\mathscr{A} \otimes \mathscr{L}, \tau_{\mathscr{B}}\right)$ and that this identification witnesses that $\left(\mathscr{B}, \tau_{\mathscr{B}}\right)$ is partitioned over $\mathscr{A}$. Therefore there exists $c \in \mathscr{L}$ are such that $\left(1 \otimes c, \tau_{\mathscr{B}}\right)$ generate an $n$-partition all of whose members are in $1 \otimes \mathscr{L}$. Let $1 \otimes c_{k}=\tau_{\mathscr{B}}^{k}(1 \otimes c) \in 1 \otimes \mathcal{L}$ for $k<n$ (or, for that matter, for all $k \in \mathbb{Z}$ ).

Let $\ell_{n}, \rho_{n}$ be as in Notation 2.10. Since $c, \ell_{n} \in \mathscr{L}$ and $\mu(c)=\mu\left(\ell_{n}\right)=\frac{1}{n}$, there is an isomorphism $\theta_{0}: \mathscr{L}_{\leq c} \cong \mathscr{L}_{\leq \ell_{n}}$, which induces in turn $\theta_{1}=\mathrm{id}_{\mathscr{A}} \otimes \theta_{0}: \mathscr{B}_{\leq 1 \otimes c} \cong$ $\mathscr{B} \leq 1 \otimes \ell_{n}$. We shall extend $\theta_{1}$ to an automorphism of $\mathscr{B}$ as follows. For $b \in \mathscr{B}$, observe that $b=\bigvee_{k<n}\left(b \wedge\left(1 \otimes c_{k}\right)\right)$ is a partition of $b$, and $\tau_{\mathscr{B}}^{-k}\left(b \wedge\left(1 \otimes c_{k}\right)\right) \in \mathscr{B}_{\leq 1 \otimes c}$. We then define

$$
\theta_{2}(b)=\bigvee_{k<n}\left(\tau_{\mathscr{A}} \otimes \rho_{n}\right)^{k} \theta_{1} \tau_{\mathscr{B}}^{-k}\left(b \wedge\left(1 \otimes c_{k}\right)\right)
$$




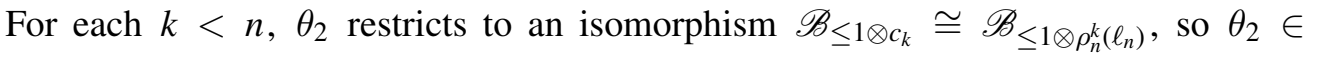
$\operatorname{Aut}(\mathscr{B})$. In addition, if $a \in \mathscr{A}$ then:

$$
\begin{aligned}
\theta_{2}\left((a \otimes 1) \wedge\left(1 \otimes c_{k}\right)\right) & =\left(\tau_{\mathscr{A}} \otimes \rho_{n}\right)^{k} \theta_{1} \tau_{\mathscr{B}}^{-k}\left(a \otimes c_{k}\right) \\
& =\left(\tau_{\mathscr{A}} \otimes \rho_{n}\right)^{k} \theta_{1}\left(\tau_{\mathscr{A}}^{-k}(a) \otimes c\right) \\
& =\left(\tau_{\mathscr{A}} \otimes \rho_{n}\right)^{k}\left(\tau_{\mathscr{A}}^{-k}(a) \otimes \ell_{n}\right) \\
& =a \otimes \rho_{n}^{k}\left(\ell_{n}\right),
\end{aligned}
$$

whereby $\theta_{2}(a \otimes 1)=a \otimes 1$. Thus $\theta_{2}$ acts as the identity on $\mathscr{A} \otimes 1$.

Let $\tau_{\mathscr{B}}^{\prime}=\theta_{2}^{-1}\left(\tau_{\mathscr{A}} \otimes \rho_{n}\right) \theta_{2}$. For $b \in \mathscr{B}$ and for $k<n$ let $b_{k}=b \wedge\left(1 \otimes c_{k}\right) \in \mathscr{B}_{\leq 1 \otimes c_{k}}$,

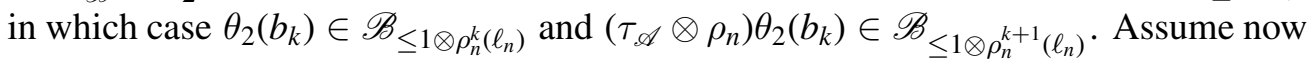
in addition that $0 \leq k \leq n-2$, i.e., that $k+1 \leq n-1$. Then in the expression $\tau_{\mathscr{B}}^{\prime}\left(b_{k}\right)=\theta_{2}^{-1}\left(\tau_{\mathscr{A}} \otimes \rho_{n}\right) \theta_{2}\left(b_{k}\right)$, the instances of $\theta_{2}^{-1}$ and $\theta_{2}$ can be expanded explicitly as follows:

$$
\begin{aligned}
\tau_{\mathscr{B}}^{\prime}\left(b_{k}\right) & =\theta_{2}^{-1}\left(\tau_{\mathscr{A}} \otimes \rho_{n}\right) \theta_{2}\left(b_{k}\right) \\
& =\left[\tau_{\mathscr{B}}^{k+1} \theta_{1}^{-1}\left(\tau_{\mathscr{A}} \otimes \rho_{n}\right)^{-k-1}\right]\left(\tau_{\mathscr{A}} \otimes \rho_{n}\right)\left[\left(\tau_{\mathscr{A}} \otimes \rho_{n}\right)^{k} \theta_{1} \tau_{\mathscr{B}}^{-k}\right]\left(b_{k}\right) \\
& =\tau_{\mathscr{B}}\left(b_{k}\right) .
\end{aligned}
$$

(On the other hand, this fails for $k=n-1$, since then $\theta_{2}^{-1}$ expands to $\theta_{1}^{-1}$.) It follows that $d\left(\tau_{\mathscr{B}}(b), \tau_{\mathscr{B}}^{\prime}(b)\right)=d\left(\tau_{\mathscr{B}}\left(b_{n-1}\right), \tau_{\mathscr{B}}^{\prime}\left(b_{n-1}\right)\right) \leq \frac{1}{2 n}$ (since both events are sub-events of same measure of $1 \otimes c_{n-1}$ which has measure $\left.\frac{1}{n}\right)$. Thus $d\left(\tau_{\mathscr{B}}, \tau_{\mathscr{B}}^{\prime}\right) \leq \frac{1}{2 n}$, as desired.

Theorem 2.12 The theory $A P r_{A}$ is $\aleph_{0}$-stable up to perturbations of the automorphism.

Proof Let $\left(\mathscr{A}, \tau_{\mathscr{A}}\right) \vDash A P r_{A}$. By Lemma $2.8\left(\mathscr{A}, \tau_{\mathscr{A}}\right)$ admits a partitioned extension $\left(\mathscr{B}, \tau_{\mathscr{B}}\right)$. We claim that up to perturbation, every type over $\mathscr{A}$ is realised in $\left(\mathscr{B}, \tau_{\mathscr{B}}\right)$.

Indeed any such type is realised in some separable extension $\left(\mathscr{C}, \tau_{\mathscr{C}}\right) \succeq\left(\mathscr{A}, \tau_{\mathscr{A}}\right)$. By Lemma 2.8 again we may assume that $\left(\mathscr{C}, \tau_{\mathscr{C}}\right)$ is also partitioned over $\mathscr{A}$. By Lemma 2.11 any two partitioned extensions of $\mathscr{A}$ admit an $\frac{1}{2 n}$ perturbation over $\mathscr{A}$ to $\left(\mathscr{A}, \tau_{\mathscr{A}}\right) \otimes\left(\mathscr{L}, \rho_{n}\right)$, and composing these we obtain an $\frac{1}{n}$-perturbation $\left(\mathscr{C}, \tau_{\mathscr{C}}\right) \rightarrow$ $\left(\mathscr{B}, \tau_{\mathscr{B}}\right)$ which fixes $\mathscr{A}$, and this for every $0<n \in \mathbb{N}$.

\section{Non superstability of probability algebras with a generic automorphism}

The second author and Henson [10] asked whether the theory of probability algebras with a generic automorphism is superstable, as one might expect by analogy with a 
theorem of Chatzidakis and Pillay [11] regarding generic automorphisms in classical first order logic. In this section we present the first author's negative answer, which chronologically came before the results in Section 2, and to a large extent motivated them.

Our aim is to show that the theory $A P r_{A}$ admits many types over small sets of parameters, and for this purpose it will suffice to show that there are many 1-types over parameters which belong to the fixed algebra of the automorphism. We therefore proceed in two steps, first characterising such types and then showing there are many of them. Throughout we let $(\mathcal{U}, \tau)$ denote a monster model of $A P r_{A}$, and let $\mathcal{U}^{\tau}$ denote the fixed sub-algebra of $\mathcal{U}$ under $\tau$.

\subsection{Types over the empty set and over fixed sub-algebras}

Let us try to describe the space of 1-types in $A P r_{A}$ over a set of parameters contained in $\mathcal{U}^{\tau}$. We start with types over the empty set.

Consider a function $\eta: 2^{<\omega} \rightarrow[0,1]$ sending $s \mapsto \eta_{s}$. We call such a function shift invariant if

$$
\eta_{\varnothing}=1, \quad \eta_{s \frown 0}+\eta_{s \frown 1}=\eta_{0 \frown s}+\eta_{1 \frown s}=\eta_{s}, \quad \text { for all } s \in 2^{<\omega} .
$$

We define $X \subseteq[0,1]^{2<\omega}$ to consist of all shift invariant mappings. This is a closed subset of $[0,1]^{2<\omega}$, and therefore compact.

Let $p \in \mathrm{S}_{1}\left(A P r_{A}\right)$, and for $n \in \mathbb{N}, s \in 2^{n}$, let $\eta_{p, s}=\mu\left(\bigwedge_{i<n} \tau^{i}\left(x^{s_{i}}\right)\right)^{p}$ (where $x^{0}=x$, $x^{1}=x^{c}$ is the natural action of $\left.(\mathbb{Z} / 2 \mathbb{Z},+)\right)$. Then $\eta_{p}: s \mapsto \eta_{p, s}$ is shift invariant, yielding a mapping $\rho: S_{1}\left(\operatorname{APr}_{A}\right) \rightarrow X$ sending $p \mapsto \eta_{p}$. This mapping $\rho$ is clearly continuous, and by quantifier elimination it is injective.

Conversely, let $\eta \in X$, and let $\mathscr{A}$ be any sufficiently homogeneous atomless probability algebra. Then one can find in $\mathscr{A}$ a sequence of events $\left(a_{n}\right)$ such that $\mu\left(a_{0}\right)=\eta_{0}$, $\mu\left(a_{0}\right)^{c}=\eta_{1}=1-\eta_{0}$, and in general, for every $n$ and $s \in 2^{n}$ :

$$
\mu\left(a_{n} \wedge \bigwedge_{k<n} a_{k}^{s_{k}}\right)=\eta_{s \frown 0}, \quad \mu\left(a_{n}^{c} \wedge \bigwedge_{k<n} a_{k}^{s_{k}}\right)=\eta_{s \frown 1}=\eta_{s}-\eta_{s \frown 0} .
$$

This is indeed consistent by shift invariance. Moreover, shift invariance implies that for every $n, k \in \mathbb{N}$ and $s \in 2^{n}: \eta_{s}=\mu\left(\bigwedge_{i<n} a_{k+i}^{s_{i}}\right)$ (by induction on $k$ ). It follows by quantifier elimination that the mapping $a_{n} \mapsto a_{n+1}$ is elementary and therefore extends to an automorphism $\tau_{\mathscr{A}} \in \operatorname{Aut}(\mathscr{A})$, and we may embed $\left(\mathscr{A}, \tau_{\mathscr{A}}\right)$ in $(\mathcal{U}, \tau)$. In other words, for every $\eta \in X$ we can find $a \in \mathcal{U}$ such that $\eta_{s}=\mu\left(\bigwedge_{i<n} \tau^{i}\left(a^{s_{i}}\right)\right)$ for all 
$s \in 2^{<\omega}$. Thus $\eta=\rho(\operatorname{tp}(a))$, showing that $\rho$ is bijective. Since it is also continuous, from a compact to a Hausdorff space, it is a homeomorphism.

If $Y$ is an arbitrary topological space we have $C(Y,[0,1])^{2^{<\omega}}=C\left(Y,[0,1]^{2^{<\omega}}\right)$ as sets. Equipping $C(Y, Z)$ with the compact-open topology and $2^{<\omega}$ with the discrete topology these are homeomorphisms. (The common topology can be given by a sub-basis, where a sub-basic open set is of the form $\left\{f \in C\left(Y \times 2^{<\omega},[0,1]\right): f[K \times\{s\}] \subseteq U\right\}$, with $K \subseteq Y$ compact, $s \in 2^{<\omega}$ and $U \subseteq[0,1]$ is open). We may define when a mapping $\eta \in C(Y,[0,1])^{2<\omega}$ is shift invariant by (SI) as above, and let $X_{Y}$ be the set of all such shift invariant functions. It is then clear that $X_{Y}=C(Y, X)$.

We now turn to types over a sub-algebra $\mathscr{A} \subseteq \mathcal{U}^{\tau}$, namely over parameters fixed by $\tau$. We shall use the following.

Fact 3.1 Let $\mathscr{A}$ be a probability algebra, and let $\widetilde{\mathscr{A}}$ be the Stone space of the underlying Boolean algebra. For an event $a \in \mathscr{A}$ let $\tilde{a} \subseteq \widetilde{\mathscr{A}}$ be the corresponding clopen set.

(i) The space $\widetilde{\mathscr{A}}$ admits a unique regular Borel probability measure $\tilde{\mu}$ such that $\tilde{\mu}(\tilde{a})=\mu(a)$ for all $a \in \mathscr{A}$.

(ii) The probability algebra of $(\widetilde{\mathscr{A}}, \tilde{\mu})$ is canonically isomorphic to $\mathscr{A}$, identifying the equivalence class of $\tilde{a}$ with the event $a$.

(iii) The natural mapping $C(\widetilde{\mathscr{A}}, \mathbb{R}) \rightarrow L_{\infty}(\widetilde{\mathscr{A}}, \tilde{\mu})$ is bijective. In other words, every equivalence class of bounded Borel functions up to equality $\tilde{\mu}$-a.e. contains a unique continuous representative.

(iv) Let $\mathscr{B} \supseteq \mathscr{A}$ be a larger probability algebra. Then there exists a conditional expectation operation $\mathbb{E}[\cdot \mid \mathscr{A}]: L_{1}(\widetilde{\mathscr{B}}) \rightarrow L_{1}(\widetilde{\mathscr{A}})$ where $\mathbb{E}[f \mid \mathscr{A}]$ is the unique function such that for all $a \in \mathscr{A}$ :

$$
\int_{\tilde{a} \subseteq \tilde{B}} f d \tilde{\mu}_{\mathscr{B}}=\int_{\tilde{a} \subseteq \tilde{A}} \mathbb{E}[f \mid \mathscr{A}] d \tilde{\mu}_{\mathscr{A}}
$$

Proof See [13]. The Stone space associated to a Boolean algebra is discussed in 311. The construction of the measure $\tilde{\mu}$ and the isomorphism between $\mathscr{A}$ and the probability algebra of $(\widetilde{\mathscr{A}}, \tilde{\mu})$ appears in 321J. The identification of $C(\widetilde{\mathscr{A}}, \mathbb{R})$ and $L_{\infty}(\widetilde{\mathscr{A}}, \tilde{\mu})$ can be found in 363 and 364K. Finally, conditional expectations are discussed in 365R.

Now let $\mathscr{A} \subseteq \mathcal{U}^{\tau} \subseteq \mathcal{U}$, and let $p \in \mathrm{S}_{1}(\mathscr{A})$, say realised by $b \in \mathcal{U}$. For $s \in 2^{n}$, the type $p$ determines the mapping associating to every $a \in \mathscr{A}$ the measure $\mu\left(a \wedge \bigwedge_{i<n} \tau^{i}\left(b^{s_{i}}\right)\right)$. In other words, $p$ determines the function $\eta_{p, s}=$ 
$\mathbb{P}\left[\bigwedge_{i<n} \tau^{i}\left(b^{s_{i}}\right) \mid \mathscr{A}\right] \in L_{1}(\widetilde{\mathscr{A}})$. Since the essential range of $\eta_{p, s}$ lies in $[0,1]$ we have $\eta_{p, s} \in L_{\infty}(\widetilde{\mathscr{A}})=C(\widetilde{\mathscr{A}}, \mathbb{R})$, and in fact $\eta_{p, s} \in C(\widetilde{\mathscr{A}},[0,1])$. Let $\eta_{p} \in C(\widetilde{\mathscr{A}},[0,1])^{2^{<\omega}}$, $\eta_{p}: s \mapsto \eta_{p, s}$. It is not difficult to see that $\eta_{p}$ satisfies (SI), so identifying $C(\widetilde{\mathscr{A}},[0,1])^{2^{<\omega}}$ with $C\left(\widetilde{\mathscr{A}},[0,1]^{2^{<\omega}}\right)$ we actually have $\eta_{p} \in X_{\mathscr{A}}:=C(\tilde{\mathscr{A}}, X)$. We have thus obtained a mapping $\rho_{\mathscr{A}}: \mathrm{S}_{1}(\mathscr{A}) \rightarrow X_{\mathscr{A}}$. Again, it is injective by quantifier elimination and continuous, and a construction as above yields that it is surjective. We have thus obtained a homeomorphism

$$
\rho_{\mathscr{A}}: \mathrm{S}_{1}(\mathscr{A}) \cong X_{\mathscr{A}}=C(\widetilde{\mathscr{A}}, X) .
$$

For a closed set $K \subseteq X$ and $a \in \mathscr{A}$ define

$$
K^{a}=\left\{\eta \in X_{\mathscr{A}}: \eta[\tilde{a}] \subseteq K\right\} .
$$

It is not difficult to see that $K^{a}$ is closed in the compact-open topology. If $\pi(x)$ is a partial type over $\varnothing$ and $K \subseteq X$ corresponds to the closed set $[\pi] \subseteq \mathrm{S}_{1}\left(A P r_{A}\right)$, then the closed set $K^{a}$ corresponds to $\left[\pi^{a}\right] \subseteq \mathrm{S}_{1}(\mathscr{A})$, where $\pi^{a}$ is a partial type over $\mathscr{A}$. If $\pi^{a}(b)$ holds we say that $b$ satisfies $\pi$ over $a$.

Let $d_{\mathscr{A}}$ denote the distance function between types over $\mathscr{A}$, and similarly $d_{\varnothing}$. It is fairly easy to verify that the distance mapping $d_{\varnothing}: \mathrm{S}_{1}\left(A P r_{A}\right)^{2} \rightarrow[0,1]$, i.e., $d_{\varnothing}: X^{2} \rightarrow$ $[0,1]$, is Borel measurable (but not continuous, since the theory is not $\aleph_{0}$-categorical). Thus, if $p, q \in \mathrm{S}_{1}(\mathscr{A})$, then $d_{\varnothing} \circ\left(\eta_{p}, \eta_{q}\right)$ is a random variable from $\mathscr{A}$ to $[0,1]$, which we can integrate.

Lemma 3.2 For all $p, q \in \mathrm{S}_{1}(\mathscr{A}): d_{\mathscr{A}}(p, q) \geq \int d_{\varnothing} \circ\left(\eta_{p}, \eta_{q}\right) d \tilde{\mu}$.

Proof Assume $a \vDash p, b \vDash q$. Let $g=\mathbb{P}[a \triangle b \mid \mathscr{A}]$. Then $g \geq d_{\varnothing} \circ\left(\eta_{p}, \eta_{q}\right) . \quad \mathbf{\square}_{3.2}$

\subsection{Non superstability proof}

We say that a continuous theory is small if the metric on $\mathrm{S}_{n}(A P r)$ is separable for all $n \in \mathbb{N}$.

Lemma 3.3 The theory $\mathrm{APr}_{A}$ is not small. More precisely, there is an uncountable family of types over the empty set every two of which have distance $\geq \frac{1}{2}$.

Proof For every real $\alpha$, let $p_{\alpha}$ be the type of one half of the circle on which $\tau$ acts by rotation by $2 \pi \alpha$ (the measure on the circle being the Lebesgue measure normalised 
to have total length 1 ). If $\alpha, \beta \geq 0$ are irrational and linearly independent over the rationals then for every $\varepsilon>0$ there exist $n, k, \ell \in \mathbb{N}$ such that $|n \alpha-k|,\left|n \beta-\ell-\frac{1}{2}\right|<\varepsilon$. If $a \vDash p_{\alpha}, b \vDash p_{\beta}$ then $d\left(a, \tau^{n}(a)\right)=\mu\left(a \triangle \tau^{n}(a)\right)<2 \varepsilon$ while $d\left(b, \tau^{n}(b)\right)>1-2 \varepsilon$. It follows that $2 d(a, b)=d(a, b)+d\left(\tau^{n}(a), \tau^{n}(b)\right) \geq 1-4 \varepsilon$, namely $d(a, b) \geq \frac{1}{2}-\varepsilon$. Therefore $d\left(p_{\alpha}, p_{\beta}\right) \geq \frac{1}{2}$ (it is not difficult to check that the distance between the types is in fact equal to $\frac{1}{2}$ ).

Let $S \subseteq \mathbb{R}$ be a vector space base for $\mathbb{R}$ over $\mathbb{Q}$. Then follows that $\left\{p_{\alpha}: \alpha \in S\right\}$ is a continuum-size set of equally distanced types.

$\mathbf{a}_{3.3}$

Proposition 3.4 There exists a family $\left\{\pi_{s}(x)\right\}_{s \in 2<\omega}$ of partial types over $\varnothing$ such that

(i) For all $s \in 2^{<\omega}$ we have $d\left(\pi_{s \frown 0}, \pi_{s \frown 1}\right) \geq \frac{1}{3}$, meaning that $d\left(a_{0}, a_{i}\right) \geq \frac{1}{3}$ whenever $a_{i} \vDash \pi_{s} \frown i$.

(ii) If $s, t \in 2^{<\omega}$ and $t$ extends $s$ then $\pi_{t} \vdash \pi_{s}$.

Proof This is a metric Cantor-Bendixson rank argument which applies more generally, saying that if $T$ is a non-small theory with a countable language then such a tree exists (with $\frac{1}{3}$ possibly replaced with another positive constant). For an even more general statement of this fact see [4, Propositions 3.16 and 3.19].

Define compacts subsets $X_{\alpha} \subseteq \mathrm{S}_{1}(\varnothing)$ by induction on $\alpha$. Start with $X_{0}=\mathrm{S}_{1}(\varnothing)$; for $\alpha$ limit, $X_{\alpha}=\bigcap_{\beta<\alpha} X_{\beta}$; and given $X_{\alpha}$, obtain $X_{\alpha+1}$ by removing from $X_{\alpha}$ all points for which there is a relatively open neighbourhood of diameter $<\frac{1}{2}$.

Since the language is countable, the topology on $X$ admits a countable base. If we only take out basic open sets of diameter $<\frac{1}{2}$ we still get the same sequence $X_{\alpha}$, and since the base is countable the sequence stabilises before $\aleph_{1}$. Let $S \subseteq \mathrm{S}_{1}(\varnothing)$ be an uncountable subset of types every pair of which have distance $\geq \frac{1}{2}$. Each set of diameter $<\frac{1}{2}$ can contain at most one member of $S$, so $X_{\aleph_{1}} \neq \varnothing$. The topological space $X_{\aleph_{1}}$ (with the induced topology) is $\frac{1}{2}$-perfect, meaning that every non-empty open subset has diameter $\geq \frac{1}{2}$.

Let $D=\left\{(q, r) \in \mathrm{S}_{1}\left(A P r_{A}\right)^{2}: d(q, r) \leq \frac{1}{3}\right\}$ and $D_{1}=D \cap X_{\aleph_{1}}^{2}$. Then $D$ is closed, being the image of the closed set $\left[d(x, y) \leq \frac{1}{3}\right] \subseteq \mathrm{S}_{2}\left(A P r_{A}\right)$ under the projection $\mathrm{S}_{2}\left(A P r_{A}\right) \rightarrow \mathrm{S}_{1}\left(A P r_{A}\right)^{2}$ (a continuous mapping from a compact space to a Hausdorff space is always closed). Thus $D_{1}$ is closed in $X_{\aleph_{1}}^{2}$.

Through the end of the proof we work in $X_{\aleph_{1}}$, with the induced topology. In particular, if $Y \subseteq X_{\aleph_{1}}$ then $Y^{\circ}$ denotes the interior of $Y$ in this topology. 
We start with $\pi_{\varnothing}(x)$ being the partial type defining $X_{\aleph_{1}}$. It has the property that $\left[\pi_{\varnothing}\right]^{\circ} \neq \varnothing$. Assume now we have $\pi_{s}$ such that $\left[\pi_{s}\right]^{\circ} \neq \varnothing$. The interior has diameter $\geq \frac{1}{2}$, so there are $q, r \in\left[\pi_{s}\right]^{\circ}$ such that $d(r, q)>\frac{1}{3}$. Thus $(q, r) \notin D_{1}$, so they admit open neighbourhoods $q \in U \subseteq\left[\pi_{s}\right]^{\circ}$ and $r \in V \subseteq\left[\pi_{s}\right]^{\circ}$ such that $(U \times V) \cap D_{1}=\varnothing$. We can then find smaller open neighbourhoods such that $q \in U_{1} \subseteq \bar{U}_{1} \subseteq U$ and $r \in V_{1} \subseteq \bar{V}_{1} \subseteq V$. Letting $\pi_{s \frown 0}$ be the partial type defining $\bar{U}_{1}$ and $\pi_{s \frown 1}$ the partial type defining $\bar{V}_{1}$ we get: $\left[\pi_{s}\right] \supseteq\left[\pi_{s \frown 0}\right] \supseteq\left[\pi_{s \frown 0}\right]^{\circ} \neq \varnothing$ and $\left[\pi_{s}\right] \supseteq\left[\pi_{s} \frown 1\right] \supseteq$ $\left[\pi_{s \frown 1}\right]^{\circ} \neq \varnothing$. Finally, $\left(\left[\pi_{s \frown 0}\right] \times\left[\pi_{s \frown 1}\right]\right) \cap D_{1}=\varnothing$ implies $d\left(\pi_{s \frown 0}, \pi_{s \frown 1}\right)>\frac{1}{3}$.

Repeating this argument we obtain the required partial types.

Lemma 3.5 The theory $\operatorname{APr}_{A} \lambda$-stable if and only if $\lambda^{\aleph_{0}}=\lambda$.

Proof One direction is since $\operatorname{APr}_{A}$ is stable in a countable language.

For the other, assume $\lambda^{\aleph_{0}}>\lambda$. Let $\left\{\pi_{s}: s \in 2^{<\omega}\right\}$ be as in Proposition 3.4. Let $\left\{a_{i}: i<\lambda\right\}$ be a sequence of independent events of measure $\frac{1}{2}$, all fixed by $\tau$, and let $\mathscr{A}$ be the generated complete algebra.

For $\theta \in \lambda^{\mathbb{N}}$ and $s \in 2^{<\omega}$ let $b_{\theta, s}=\bigwedge_{i<|s|} a_{\theta(i)}^{s(i)} \in \mathscr{A}$, and let

$$
\rho_{\theta, n}=\bigcup_{s \in 2^{n}} \pi_{s}^{b_{\theta, s}}(x), \quad \rho_{\theta}=\bigcup_{n} \rho_{\theta, n} .
$$

In other words, $\rho_{\theta}(c)$ holds if and only if, for every $s \in 2^{<\omega}, c$ satisfies $\pi_{s}$ over $\bigwedge_{i<|s|} a_{\theta(i)}^{s(i)}$. It is easy to check that $\rho_{\theta, n}$ is consistent and implies $\rho_{\theta, m}$ for $m<n$, so $\rho_{\theta}$ is consistent as well. Choose for each $\theta$ a complete type $r_{\theta} \in \mathrm{S}_{1}(\mathscr{A})$ extending $\rho_{\theta}$.

Let $\theta \neq \theta^{\prime} \in \lambda^{\mathbb{N}}$, and let $i \in \mathbb{N}$ be such that $\theta(i) \neq \theta^{\prime}(i)$. Then over $a_{\theta(i)} \backslash a_{\theta^{\prime}(i)}$, $\eta_{r_{\theta}}$ takes only values in $\bigcup_{s \in 2^{i}}\left[\pi_{s} \frown 0\right] \subseteq \mathrm{S}_{1}\left(\operatorname{APr}_{A}\right)$, while $\eta_{r_{\theta^{\prime}}}$ only takes values in $\bigcup_{s \in 2^{i}}\left[\pi_{s \frown 1}\right]$, and the opposite holds over $a_{\theta^{\prime}(i)} \backslash a_{\theta(i)}$. Thus $d_{\varnothing} \circ\left(\eta_{r_{\theta}}, \eta_{r_{\theta^{\prime}}}\right) \geq \frac{1}{3}$ over $a_{\theta(i)} \triangle a_{\theta^{\prime}(i)}$, which has measure $\frac{1}{2}$. We conclude that $d\left(r_{\theta}, r_{\theta^{\prime}}\right) \geq \frac{1}{6}$.

We have shown that there are $\lambda^{\mathbb{N}}$ equally distanced types over a set of $\lambda$ parameters, as desired.

We conclude:

Theorem 3.6 The theory $\mathrm{APr}_{A}$ is not superstable, and therefore not supersimple.

Notice the difference from the case of $I H S_{A}$, which is superstable (but not $\aleph_{0}$-stable). 


\section{References}

[1] I Ben Yaacov, On perturbations of continuous structures, arXiv:0802.4388, submitted.

[2] I Ben Yaacov, Uncountable dense categoricity in cats, Journal of Symbolic Logic 70 (2005) 829-860; doi:10.2178/jsl/1122038916.

[3] I Ben Yaacov, On supersimplicity and lovely pairs of cats, Journal of Symbolic Logic 71 (2006) 763-776; doi:10.2178/jsl/1154698575 ; arXiv:0902.0118.

[4] I Ben Yaacov, Topometric spaces and perturbations of metric structures, Logic and Analysis 1 (2008), 235-272; doi: 10.1007/s11813-008-0009-x; arXiv:0802.4458.

[5] I Ben Yaacov, Modular functionals and perturbations of Nakano spaces, Journal of Logic and Analysis 1 (2009), 1-42; doi: 10.4115/jla.2009.1.1; arXiv:0802.4285.

[6] I Ben Yaacov, A Berenstein, C W Henson, A Usvyatsov, Model theory for metric structures, in: Model theory with Applications to Algebra and Analysis, Vol. 2, (Z Chatzidakis, D Macpherson, A Pillay, A Wilkie, editors), London Math Society Lecture Note Series 350 (2008), Cambridge University Press, 315-427.

[7] I Ben Yaacov, A Usvyatsov, Continuous first order logic and local stability, Transactions of the American Mathematical Society, to appear; arXiv:0801.4303.

[8] I Ben Yaacov, A Usvyatsov, M Zadka, Hilbert spaces with a generic automorphism, in preparation.

[9] A Berenstein, Hilbert spaces with generic groups of automorphisms, Archive for Mathematical Logic 46 (2007), 289-299; doi:10.1007/s00153-007-0044-4.

[10] A Berenstein, C W Henson, Model theory of probability spaces with an automorphism, submitted.

[11] Z Chatzidakis, A Pillay, Generic structures and simple theories, Annals of Pure and Applied Logic 95 (1998), 71-92; doi: 10.1016/S0168-0072(98)00021-9.

[12] K R Davidson, $C^{*}$-algebras by example, Fields Institute Monographs Vol. 6, American Mathematical Society, Providence, RI (1996).

[13] D H Fremlin,

Measure Theory Volume 3: Measure Algebras, Torres Fremlin, 25 Ireton Road, Colchester CO3 3AT, England (2004), http://www.essex.ac.uk/maths/staff/fremlin/mt3.2004/index.htm.

[14] J Iovino, Stable Banach spaces and Banach space structures, I and II, in: Models, Algebras, and Proofs: Selected Papers of the X Latin American Symposium on Mathematical Logic Held in Bogotá 1996 (Xavier Caicedo, Carlos H. Montenegro, editors), 1996, Lecture Notes in Pure and Appl. Math. 203, Dekker, New York (1998), 77-117. 
Itaï Ben Yaacov, Université Claude Bernard - Lyon 1, Institut Camille Jordan, 43 boulevard du 11 novembre 1918, 69622 Villeurbanne Cedex, France

Alexander Berenstein, Universidad de los Andes, Departamento de Matemticas, Carrera 1 \# 18A-10, Bogotá, Colombia ; and

Université Claude Bernard - Lyon 1, Institut Camille Jordan, 43 boulevard du 11 novembre 1918, 69622 Villeurbanne Cedex, France

aberenst@uniandes.edu.co

http://math.univ-lyon1.fr/ begnac,

http://matematicas.uniandes.edu.co/ aberenst

Received: 20 October 2008 Revised: 2 June 2009 